\title{
Clima social familiar e impacto en el rendimiento académico de los estudiantes
}

\section{Family social climate and impact on the academic performance of students}

\author{
Clima social familiar e impacto no desempenho acadêmico dos estudantes
}

Juliana Paola Méndez- Omaña ${ }^{a *}$, Luis Alberto Jaimes- Contreras ${ }^{\mathrm{b}}$

${ }^{a}$ Máster Universitario en Neuropsicología y educación, Universidad de Pamplona (Cúcuta). (D)

${ }^{\mathrm{b}}$ Magíster en docencia de la Matemática, Universidad Francisco de Paula Santander, Cúcuta-Colombia. (D)

Forma de citar: Méndez, J. \& Jaimes, L. (2018). Clima Social familiar e impacto en el rendimiento académico de los estudiantes. Perspectivas, 3(1). 24-43

Recibido: julio 10 de 2017

Aceptado: octubre 05 de 2017

\section{Palabras clave}

Clima social familiar, familia, rendimiento académico.

* Autor para correspondencia

juliana.mendez@unipamplona.edu.co

https://doi.org/10.22463/25909215.1422 


\section{Keywords}

Family social climate, family, academic performance.

\section{Palavras chave}

Clima social familiar, família, desempenho acadêmico.

\begin{abstract}
The main objective of the research presented was to establish the relationship between the family social climate and academic performance in a group of students from a public institution and a private one from the city of Bogotá. To carry out this study, the quantitative methodology with a cross-sectional design was used, where 49 students of both genders were evaluated, between the ages of 13 and 17 who are in the eighth grade, to whom the social climate scale was applied. family of Moos. The results of the investigation showed that in none of the institutions is a degree of association between academic performance and the family social climate being presented, however, in the discussion other possible factors to be considered in the research are cited.
\end{abstract}

\begin{abstract}
Resumo: O objetivo principal da pesquisa apresentada foi estabelecer a relação entre o clima social familiar e o desempenho acadêmico em um grupo de estudantes de uma instituição pública e um particular da cidade de Bogotá. Para realizar este estudo, trabalhamos na metodologia quantitativa, com delineamento transversal, onde foram avaliados 49 estudantes de ambos os sexos, com idades entre 13 e 17 anos, da oitava série, aos quais foi aplicada a escala climática social e familiar. de Moos. Os resultados da investigação mostraram que em nenhuma das instituições existe um grau de associação entre o desempenho acadêmico e o clima social familiar apresentado, entretanto, na discussão, outros possíveis fatores a serem considerados na pesquisa são citados.
\end{abstract}

\section{Introducción}

Dentro de la sociedad colombiana se ha identificado como una problemática el bajo rendimiento académico, esta dificultad de índole educativo afecta al desarrollo de la sociedad, por lo tanto, se está generando un índice elevado de conflictos a nivel social; pero a su vez las entidades buscan dar soluciones de manera mediática al problema sin indagar en posibles causas o determinar la relación del estudiante en su ambiente escolar con lo personal y familiar. Po $\mathrm{r}$ ello, es relevante la necesidad de realizar investigaciones relacionadas con dicha temática y plantearse interrogantes que permitan llegar a información útil para la efectiva evaluación e intervención de los profesionales del área de salud mental y social.
Según García (2005), refiere que el clima social familiar está relacionado con las interacciones que los padres desarrollan con los hijos en el hogar, estas pueden variar en cantidad y calidad. Como se sabe, el tipo de interacción familiar que establecen los sujetos desde su temprana infancia ejerce influencia en sus diferentes etapas de vida, facilitando o dificultando las relaciones en las diferentes esferas de actividad: educativa, formativa, social y familiar.

Desde esta óptica, la interacción de padres e hijos es de vital importancia para el desarrollo de las diversas esferas que rodean al individuo, ya que de una u otra manera ejercen gran influencia en el rendimiento académico de los adolescentes.

"Partiendo de esta premisa las actitudes y conductas que presentan las personas son producto de un proceso de condicionamiento y aprendizaje 
producido en el ambiente familiar. Se encuentra una amplia gama de estudios desarrollados acerca de la influencia del clima social familiar en el rendimiento académico", Rojas (2005), González y Pereda (2006), Torres y Rodríguez (2006), Adue(1997), Sánchez y Valdés (2011). Es precisamente por esto que el presente trabajo analiza la repercusión que tiene la familia en las relaciones interpersonales, específicamente ante las situaciones de agravio como lo es el rendimiento académico de los adolescentes.

De acuerdo a lo anterior, se pretendió determinar la relación existente del clima social familiar y el rendimiento académico de los estudiantes de una institución pública y una institución privada de la ciudad de Bogotá, que se encuentran en un rango de 13 y 17 años de edad, de grado octavo, en el cual se abordaran temáticas como el rendimiento académico en relación al clima social familiar, se hará mediante la aplicación del test de Moos y se correlacionara con el rendimiento académico de los estudiantes. Asimismo, cabe resaltar la importancia de esta investigación, que radica en la importancia de la familia en el acompañamiento escolar con el propósito de fortalecer el rendimiento académico de los estudiantes.

El fracaso escolar es un fenómeno multifactorial de gran complejidad donde inciden diversidad de variables personales, socio familiar y académicas. Aunque han sido especialmente frecuentes los estudios de los aspectos personales y académicos del fracaso escolar, se olvida continuamente el entorno social que rodea al estudiante y que ejerce un significativo papel en la vida académica de los estudiantes, tanto directa como indirectamente.

Prosiguiendo con el tema, uno de los factores que pueden afectar el rendimiento académico son los problemas en la familia, ya que suelen influir en el comportamiento de los niños, en su conducta escolar y en su rendimiento académico. La falta de atención de los padres, el exceso de privilegios, la falta de autoridad o la violencia intrafamiliar pueden ser las causas del fracaso escolar.
Por otra parte, diversos estudios (Anabalon etal; 2008, Baeza, 2000 , 2006) "ostentan que la familia forma parte de los agentes que van a intervenir en el desarrollo de habilidades académicas; asimismo se encuentran las diversas situaciones que se desencadenan dentro del ambiente familiar, todo esto con el fin de conocer si los padres realmente se involucran con el desarrollo de sus hijos".

En este orden de ideas, es importante tener en cuenta el ambiente familiar, ya que cada individuo comienza a cimentar su carácter y su personalidad, a través de los padres que son las personas más cercanas y se encargan del cuidado y la protección de cada uno de los miembros que integran la familia, de igual manera son los encargados de disciplinar conductas por medio de normas, límites y reforzadores positivos. "El entorno familiar es de suma importancia, ya que son parte del constructo de perturbaciones en el aprendizaje" (Bima; 1980), dichas perturbaciones se ven reflejadas en el desempeño escolar, es por esta razón que se intentara confirmar si esta problemática está afectando el rendimiento de la población a trabajar.

Sin embargo, conviene advertir, que las relaciones interpersonales juegan un papel primordial en el desarrollo integral del individuo. Por medio de ellas, la persona alcanza importantes refuerzos sociales del entorno más inmediato que favorecen su adaptación al mismo. En contrapartida, la falta de estas habilidades puede estimular rechazo, aislamiento y, en definitiva, limitar la calidad de vida del individuo.

Por otra parte, los adolescentes de hoy en día pasan más tiempo fuera de casa y disminuye la cantidad de interacciones positivas con los padres, incrementando los conflictos relacionados con aspectos de la vida cotidiana tales como las labores de la casa, amistades, la forma de vestir o la hora de llegada a casa.

Según García (2005) "nos refiere que el clima social familiar está relacionado con las interacciones que los Padres desarrollan con los hijos en el hogar, estas pueden variar en cantidad y calidad". Como se sabe, el tipo de interacción familiar 
que establecen los sujetos desde su temprana infancia ejerce influencia en sus diferentes etapas de vida, facilitando o dificultando las relaciones en las diferentes esferas de actividad: educativa, formativa, social y familiar.

Por otra parte, se ha logrado catalogar a la familia como núcleo de la sociedad, por lo tanto, tal denominación amerita algún tipo de investigación, en este caso la relación del clima social familiar y el rendimiento académico de los estudiantes, ya que hay que tener en cuenta que como individuo se forma parte de una familia, en esta medida la información adquirida influirá de una u otra manera en su ámbito educativo.

A partir de lo anterior resulta evidente el valor de la familia como principal entorno de socialización y recurso fundamental para los niveles de logro en otros contextos tales como la escuela. "Si bien el status socioeconómico de la familia es importante para la formación de los niños, este factor no resulta decisivo; más decisivos resultan ser la organización de la familia, su clima afectivo, la adquisición temprana de actitudes y motivaciones y la comunicación familia -escuela, el involucramiento de la familia en las tareas escolares, la articulación entre los códigos culturales de la familia y escuela" (Barudy y Dantagnan, 2005).

Por lo tanto, una buena conjunción de esfuerzos de ambas instituciones familia y escuela, sería la pieza clave de una pedagogía efectiva. "De hecho, algunas experiencias de investigación- intervención muestran que sólo es posible cambiar percepciones, actitudes y comportamientos de padres y educadores a través de una intensificación de las relaciones de trabajo y de comunicación entre ambos" (CIDE \& UNICEF, 2000; González, 1999).

Rendimiento Académico. Se discurre que el rendimiento académico es el nivel de conocimientos estudiados de un área o materia comparada con la norma (edad, nivel académico). Así, tal rendimiento no es sinónimo de capacidad intelectual, de aptitudes o competencias.
En la mayor parte de la literatura sobre el rendimiento escolar hay estudios sobre los factores asociados al fracaso escolar; sin embargo, son esos mismos factores son los que propician también el éxito escolar.

Según Papalia, Wendkos y Dustin (2005), Perez (1996); Polaino (2004), consideran que "parece existir una aprobación de que la lista de las causas del fracaso o del éxito escolar es amplia, ya que va desde lo personal hasta lo sociocultural, haciendo la mayoría de las veces una mezcla tanto de factores personales como sociales" (p. 18).

La teoría del clima social de Moos. Escala de Clima Social en la Familia (Moos, 1974; Moos y Trickett, 1974): Evalúa las relaciones interpersonales entre los miembros de la familia, la dirección marcada desde el seno familiar para el crecimiento personal, y su estructura organizativa básica (Moss y Moos, 1981). Se ha utilizado la versión validada en español (Moos, Moos y Trickett, 1995), citado por de (Seis de dos, Victoria de la Cruz y Cordero, 1989 ; Dios, C. de et al, 2003).

En este punto, es importante resaltar que Moos (1974) definió el clima social como la personalidad del ambiente en base a las percepciones que los habitantes tienen de un determinado ambiente yentre las que figuran distintas dimensiones relacionales. "Así una determinada clase de un centro escolar puede ser más o menos creativa, afiliativa, orientada a las tareas, etc.

Asimismo, una específica familia puede ser más o menos controladora, cohesiva, organizada, etc. Según Fernández-Ballesteros y Sierra (1982), el objetivo de Moos y sus colegas ha sido encontrar invariantes de tales atributos a través de diferentes ambientes sociales.

Según Cassullo, Álvarez y Pasman, (1998 ), el análisis del contexto es de excepcional importancia en el estudio de la conducta de un sujeto, tanto para caracterizar la conducta en sí misma como para captar el valor de significación que tal conducta toma en el grupo de social de referencia, por lo tanto, el clima social es una de las más importantes 
conceptualizaciones en el estudio de la conducta humana en contextos sociales.

Por otra parte, García (2005), refiere que el clima familiar está relacionado con las interacciones que los padres desarrollan con los hijos en el hogar. Éstas pueden variar en cantidad y calidad. Para este autor, el tipo de interacción familiar que establecen los individuos desde su temprana infancia ejerce influencia en las diversas etapas de la vida, lo que facilita o dificulta las relaciones en las distintas esferas de actividad: educativa y formativa.

Al respecto conviene citar a Moreno, Estévez, Murgui y Musitu (2009) quienes sostienen que el clima familiar es uno de los factores de mayor relevancia en el ajuste psicosocial de los hijos; ejerce una influencia significativa tanto en la conducta, como el desarrollo social, físico, afectivo e intelectual de los integrantes.

En este punto cabe citar a Musitu y García, (2004 ), quienes afirman que un clima familiar positivo se fundamenta en la cohesión afectiva entre padres e hijos, el apoyo, la confianza e intimidad, los cuales potencian el ajuste conductual y psicológico de los hijos.

En este orden de ideas y de acuerdo con Kemper, S. (2000) quien sostiene que el clima social que es un concepto y cuya operacionalización resulta difícil de universalizar, pretende describir las características psicológicas e institucionales de un determinado grupo humano situado sobre un ambiente. Citando a Moos (1974), manifiesta que para estudiar o evaluar el clima social familiar, son tres las dimensiones o atributos afectivos que hay que tener en cuenta, para lo cual ha elaborado diversas escalas de Clima Social aplicables a diferentes tipos de ambiente, como es el caso de la Escala del Clima Social en la Familia (FES).

En ella se trata sobre las interrelaciones que se dan entre los miembros de la familia donde se cumplen las funciones de comunicación, interacción. El desarrollo personal puede ser fomentado por la vida en común, así como la organización y el grado de control que se ejercen unos miembros sobre otros.
Dimensiones y áreas del clima social familiar. Las dimensiones que evalúa:

- Actuación (AC): Grado en el que las actividades (tales como el Colegio o el Trabajo) se enmarcan en una estructura orientada a la accióncompetencia.

- Autonomía (AU): Grado en el que los miembros de la familia están seguros de sí mismos, son independientes y toman sus propias decisiones.

- Cohesión (CO): Mide el grado en el que los miembros del grupo familiar están compenetrados y se apoyan entre sí.

- Conflictos (CT): Grado en el que se expresan libre y abiertamente la cólera, agresividad y conflicto entre los miembros de la familia.

- Control(CN): Grado en el que la dirección de la vida familiar se atiene a reglas y procedimientos establecidos.

- Desarrollo: evalúa la importancia que tiene dentro de la familia, ciertos procesos de desarrollo personal, que pueden ser fomentados o no, por la vida en común. Esta dimensión está integrada por las siguientes áreas.

- Estabilidad: proporciona información sobre la estructura y organización de la familia y sobre el grado de control que normalmente ejercen unos miembros de la familia sobre otros, integrada por las áreas:

- Expresividad (EX): Explora el grado en el que se permite y anima a los miembros de la familia a actuar libremente y a expresar directamente sus sentimientos.

- Intelectual - cultural: Grado de interés en las actividades de tipo Político, Intelectual, Cultural y social.

- Moralidad - religiosidad: Importancia que se le da a las prácticas y valores de tipo ético y religioso.

- Organización (OR): Importancia que se le da en el hogar a una clara organización y estructura al planificar las actividades y responsabilidades de la familia. 
- Relaciones: Mide el grado de comunicación y libre expresión al interior de la familia y el grado de interacción conflictiva que la caracteriza, integrada por las siguientes áreas.

- Social-Recreativo (SR): Grado de participación en este tipo de actividades.

\section{Metodología}

\section{Tipo de investigación}

El estudio ha realizado es de carácter cuantitativo. Por otra parte, el diseño es de tipo transversal correlacional - causal, que intenta conocer la relación existente entre el clima social familiar y el rendimiento académico de los estudiantes y a su vez comparar los resultados obtenidos en las dos instituciones.

La investigación trasversal correlacional causal consiste, en describir relaciones entre dos o más categorías, conceptos o variables en un momento determinado ya sea en términos correlaciónales o en función de la relación causa- efecto.

\section{Población}

La población estuvo conformada por un grupo de estudiantes de una institución pública y una privada de la ciudad de Bogotá. Un grupo de 40 estudiantes de grado octavo de la institución educativa publica Alfonzo López Michels en jornada de la tarde, sus edades oscilan entre los 13 y 17 años, pertenecen a un nivel socioeconómico medio bajo. Por otra parte, la institución educativa privada Liceo Moderno Celestin Freinet se encuentra conformada por un grupo de 11 estudiantes de grado octavo de la jornada de la mañana sus edades oscilan entre los 13 y 16 años, pertenecen a un nivel socioeconómico medio bajo.

\section{Muestra.}

El tipo de muestra es no probabilística, dado que la elección de los elementos no depende de la probabilidad, sino de causas relacionada con las características de la investigación. La muestra representativa consta de 50 adolescentes, conformado por mujeres y hombres, sus edades oscilan entre 13 y 16 años que se encuentran cursando octavo grado y presentan dificultades en el rendimiento académico de las instituciones educativas pública y privada de la ciudad de Bogotá.

\section{Hipótesis de investigación}

Hi: Existe relación significativa del clima social familiar y el rendimiento académico en los estudiantes de la institución educativa pública y privada.

H0: No Existe relación significativa del clima social familiar y el rendimiento académico en los estudiantes de la institución educativa pública y privada.

\section{Hipótesisalternativas.}

Ha: La categoría de las instituciones (públicoprivada),es influyente en el rendimiento y clima social familiar.

\section{Instrumentos para la recolección de datos}

Se utilizo el "Cuestionario del Test de Moos", para medir el Clima Socio Familiar de los estudiantes de la Institución Educativa Alfonso López Michels en y la Institución Educativa Liceo Moderno Celestin Freinet de la ciudad de Bogotá

\section{Ficha Técnica}

- Nombre del Instrumento: Escala del Clima Socio Familiar

- Autores: R.H. Moos. y E.J. Trickett

- Estandarización: Ana Alegre Haro - Yahel QuirozMejía - Recuay - 2010

- Administración: Individual. Tiempo Aplicación: 90 minutos.

- Significación: Evalúa las características socio ambientales y las relaciones personales en la familia 
Las actas de evaluación permitieron obtener datos acerca de las calificaciones del rendimiento académico de los estudiantes que constituyen la muestra del presente estudio, correspondiente al año 2012

\section{Interpretación de los resultados.}

Se realizo la interpretación de los resultados obtenidos en la clase de correlación entre las variables evaluadas, desde una perspectiva integradora de estos y en función de los objetivos propuestos en esta investigación, como punto de partida tendremos un planteamiento de la Psicología ambiental que señala Holaban (1996); citado por Kemper, S.(2000). Que la Psicología Ambiental comprende una amplia área de investigación relacionada con los efectos psicológicos del ambiente y su influencia sobre el individuo.

Por otra parte, Moos (1974), definió el clima social como la personalidad del ambiente en base a las percepciones que los habitantes tienen de un determinado ambiente y entre las que figuran distintas dimensiones relacionales.

Debido a lo anterior se desgloso los resultados para dar a entender algunas generalidades $\mathrm{y}$ particularidades encontradas en el proceso, así mismo la aceptación o negación de hipótesis. Primeramente, es importante aclarar que, para el análisis de correlación para la institución pública, se utilizó el modelo de regresión logística que permite estudiar si una variable binomial depende, o no, de otra u otras variables (no necesariamente binomiales). La variable respuesta binaria de interés en este caso es el CSF con dos posibles resultados: Adecuado e Inadecuado, y la variable independiente es el rendimiento académico.

Por el contrario, con la institución educativa privada se aplicó un modelo diferente partiendo de que la muestra es más pequeña, en este caso se utilizó el test exacto de Fisher, que permite analizar si dos variables dicotómicas están asociadas cuando la muestra a estudiar es demasiado pequeña y no se cumplen las condiciones necesarias para que la aplicación del test chi-cuadrado sea adecuada.
Para iniciar partiremos de la negación de la hipótesis central ya que la media probabilística nos indica que no existe correlación entre las variables bajo rendimiento y clima social familiar en las dos instituciones trabajadas, dado que el porcentaje de correlación es de $0.269 \%$, lo cual no es significativo para indicar un grado de correlación; según los planteamientos de Moos, de la psicología ambiental donde demarca que existen otros factores que pueden relacionarse con este fenómeno, como lo es el clima escolar que puede ser un factor influyente en el rendimiento académico, partiendo de esta premisa plantea una escala en donde se estudia la relación docente - estudiante "escala del clima social escolar".

Sin embargo cabe resaltar que en la categorización de rendimiento académico no se encontró un porcentaje alto de estudiantes en nivel bajo pero si en su mayoría en básico lo que serelaciona en el caso de la institución pública se desataca que de los casos inadecuados detectados el $58.8 \%$ de estos tiene relación con un rendimiento escolar Básico, el cual no se denomina como destacado, entonces se puede decir que en su mayoría puede obstaculizar el potencializar al joven en su proceso formativo como afirman Pelegrina, Linares, Casanova (2001), quienes consideran que un ambiente familiar que estimule el placer por las tareas académicas, la curiosidad por el saber, la persistencia por el logro académico se relaciona con resultados académicos buenos.

Por otro lado, se pudo establecer según los resultados que al realizar la comparación entre las dos instituciones estas disciernen en lo referente a la correlación de los casos inadecuados con el nivel de rendimiento y a la presencia de estos, ya que en la privada solo existe un caso que corresponde al 10\% de la población y este es en nivel superior por el contrario en el público los casos de inadecuado son el $43.6 \%$ y se ubican en las diferentes categorías de rendimiento escolar.

En cuanto a la hipótesis alternativa donde se pretendió corroborar si existe influencia entre las variables analizadas y que sea una institución 
pública o privada se puede determinar que según los resultados porcentuales esto es influyente ya que desde el mismo índice de casos existentes y las categorías de rendimiento escolar ya que en la institución pública se evidencian casos de rendimiento académico bajo, mientras que el privado no existe ni un solo caso en rendimiento escolar bajo.

Por otra parte, se observó mediante la aplicación del test Moos (ECF), que los estudiantes de la institución privada contaron con mayor disposición a la hora de cumplir las normas establecidas por el instructor de la prueba, mientras que por parte de la institución pública se evidencio dificultad la hora de identificarse en el test a pesar de haber iniciado la aplicación de la prueba a partir de confidencialidad.

\section{Resultados}

A continuación, se hace una presentación de la categorización del rendimiento escolar de la institución educativa pública y privada.
Nivel académico

Superior

Alto

Básico

Bajo
Promedio

$4.5-5.0$

$4.0-4.5$

3.5-4.0

Menores a 3.5

Tabla 2

Categorización del Rendimiento Escolar del Colegio Privado.

Tabla de categorización del rendimiento escolar (privado)

Nivel académico

Superior

Alto

Básico

Bajo
Promedio

4.8-5.0

$4.0-4.7$

3.0-3.9

$0.0-2: 9$ 
Figura 1

Rendimiento académico de las dos instituciones.

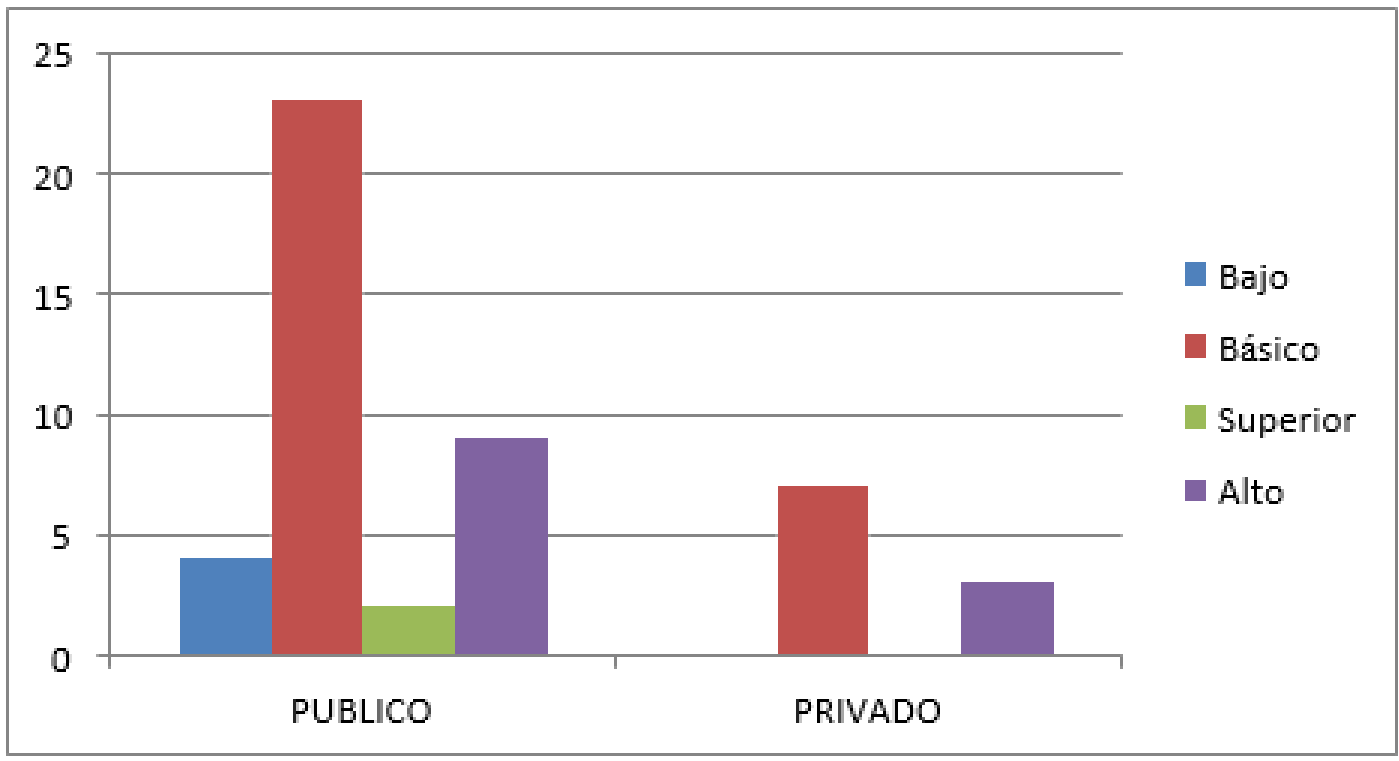

Estudio sobre el clima social familiar (CSF) pública. A continuación, se presenta la información y el rendimiento académico en la institución recolectada a través de cuestionario test de Moss (CSF).

Cuadro 1.

Datos para el clima social familiar y el rendimiento académico colegio público.

\begin{tabular}{|cccc|l|l|}
\hline ID & DR & DD & DE & CSF & RENDIMIENTO \\
\hline 8 & 13 & 26 & 13 & ADECUADO & BAJO \\
4 & 14 & 25 & 8 & INADECUADO & BAJO \\
29 & 12 & 24 & 14 & ADECUADO & BAJO \\
32 & 16 & 32 & 12 & ADECUADO & BAJO \\
39 & 13 & 22 & 15 & ADECUADO & BAJO \\
3 & 15 & 25 & 12 & ADECUADO & BÁSICO \\
5 & 17 & 33 & 15 & ADECUADO & BÁSICO \\
6 & 10 & 29 & 13 & ADECUADO & BÁSICO \\
25 & 16 & 29 & 14 & ADECUADO & BÁSICO \\
34 & 17 & 20 & 13 & ADECUADO & BÁSICO \\
37 & 13 & 26 & 11 & ADECUADO & BÁSICO \\
7 & 13 & 18 & 11 & INADECUADO & BÁSICO \\
11 & 13 & 22 & 13 & INADECUADO & BÁSICO \\
13 & 15 & 33 & 9 & ADECUADO & BÁSICO \\
21 & 14 & 23 & 11 & INADECUADO & BÁSICO \\
12 & 13 & 29 & 14 & ADECUADO & BÁSICO
\end{tabular}




\begin{tabular}{|c|c|c|c|c|c|}
\hline 14 & 13 & 24 & 12 & INADECUADO & BASICO \\
\hline 18 & 12 & 21 & 11 & INADECUADO & BÁSICO \\
\hline 22 & 14 & 21 & 11 & INADECUADO & BÁSICO \\
\hline 15 & 13 & 16 & 13 & INADECUADO & BÁSICO \\
\hline 20 & 16 & 35 & 12 & ADECUADO & BÁSICO \\
\hline 24 & 13 & 25 & 10 & INADECUADO & BÁSICO \\
\hline 28 & 13 & 21 & 9 & INADECUADO & BÁSICO \\
\hline 36 & 6 & 18 & 15 & INADECUADO & BÁSICO \\
\hline 16 & 12 & 28 & 13 & ADECUADO & BÁSICO \\
\hline 19 & 12 & 27 & 14 & ADECUADO & BÁSICO \\
\hline 26 & 15 & 35 & 12 & ADECUADO & BÁSICO \\
\hline 35 & 15 & 26 & 10 & ADECUADO & BÁSICO \\
\hline 1 & 11 & 25 & 11 & INADECUADO & ALTO \\
\hline 2 & 16 & 31 & 11 & ADECUADO & ALTO \\
\hline 38 & 15 & 25 & 7 & INADECUADO & ALTO \\
\hline 10 & 18 & 21 & 14 & ADECUADO & ALTO \\
\hline 17 & 15 & 31 & 14 & ADECUADO & ALTO \\
\hline 30 & 15 & 26 & 15 & ADECUADO & ALTO \\
\hline 31 & 18 & 26 & 13 & ADECUADO & ALTO \\
\hline 9 & 17 & 18 & 13 & INADECUADO & ALTO \\
\hline 27 & 12 & 23 & 14 & INADECUADO & ALTO \\
\hline 23 & 13 & 19 & 12 & INADECUADO & SUPERIOR \\
\hline 33 & 12 & 21 & 12 & INADECUADO & SUPERIOR \\
\hline
\end{tabular}

Las variables consideradas son Dimensión de relaciones (DR), Dimensión de desarrollo (DD), Dimensión de estabilidad (DE), a partir de las cuales se calcula elCSF y finalmente la variable rendimiento académico, que se denota por "rendimiento".
Análisis Estadístico: Las variables del cuadro 1 CSF y Rendimiento se analizarán con ayuda de una tabla de contingencias y para probar si hay relación entre estas dos variables se utilizará un modelo lineal generalizado.

\section{Análisis descriptivo:}

Cuadro 2. Contingencias CSF* Rendimiento.

\begin{tabular}{|c|c|c|c|c|c|c|}
\hline & \multicolumn{4}{|c|}{ RENDIMIENTO } & \multirow[b]{2}{*}{ Total } \\
\hline & & ALTO & BAJO & BÁSICO & SUPERIOR & \\
\hline \multirow[t]{2}{*}{ CSF } & ADECUADO & 5 & 4 & 13 & 0 & 22 \\
\hline & INADECUADO & 4 & 1 & 10 & 2 & 17 \\
\hline \multicolumn{2}{|l|}{ Total } & 9 & 5 & 23 & 2 & 39 \\
\hline
\end{tabular}


El cuadro 2 ofrece las frecuencias de cruzar el clima social familiar y el rendimiento académico. Así, sabemos que 56,4\% de las personas encuestadas pertenecen a un CSF adecuado y el restante 43.6\% a un CSF inadecuado. Adicionalmente, se observa que el rendimiento académico más frecuente es el básico con una representatividad del $59 \%$ sobre el total de los estudiantes, mientras que el superior es el menos frecuente con el 5.1\%. De igual manera, se observa que ningún estudiante tiene un CSF adecuado y a su vez un rendimiento académico superior.

Cuadro 3.

Frecuencias marginales CSF * Rendimiento.

\begin{tabular}{|c|c|c|c|c|c|c|}
\hline & \multirow{2}{*}{ CSF } & \multicolumn{4}{|c|}{ RENDIMIENTO } & \multirow[b]{2}{*}{ Total } \\
\hline & & ALTO & BANO & BÁSICO & SUPERIOR & \\
\hline \multirow[t]{4}{*}{ ADECUADO } & Frecuencia & 5 & 4 & 13 & 0 & 22 \\
\hline & $\%$ dentro de CSF & $22,7 \%$ & $18,2 \%$ & $59,1 \%$ & $.0 \%$ & $100,0 \%$ \\
\hline & $\%$ dentro de RENDIMIENTO & $55,6 \%$ & $80.0 \%$ & $56,5 \%$ & $.0 \%$ & $56,4 \%$ \\
\hline & $\%$ del total & $12,8 \%$ & $10.3 \%$ & $33,3 \%$ & $.0 \%$ & $56,4 \%$ \\
\hline \multirow[t]{4}{*}{ INADECUADO } & Frecuencia & 4 & 1 & 10 & 2 & 17 \\
\hline & $\%$ dentre de CSF & $23,5 \%$ & $5,9 \%$ & $58,8 \%$ & $11,8 \%$ & $100,0 \%$ \\
\hline & $\%$ dentro de RENDIMIENTO & $44,4 \%$ & $20,0 \%$ & $43,5 \%$ & $100,0 \%$ & $43,6 \%$ \\
\hline & $\%$ del total & $10.3 \%$ & $2.6 \%$ & $25,6 \%$ & $5,1 \%$ & $43.6 \%$ \\
\hline \multirow[t]{4}{*}{ Total } & Frecuencia & 9 & 5 & 23 & 2 & 39 \\
\hline & $\%$ dentre de CSF & $23,1 \%$ & $12,8 \%$ & $59,0 \%$ & $5,1 \%$ & $100,0 \%$ \\
\hline & \% dentro de RENDIMIENTO & $100,0 \%$ & $100,0 \%$ & $100,0 \%$ & $100,0 \%$ & $100,0 \%$ \\
\hline & $\%$ del total & $23,1 \%$ & $12,8 \%$ & $59,0 \%$ & $5,1 \%$ & $100,0 \%$ \\
\hline
\end{tabular}

El cuadro 3 También ofrece los totales correspondientes a cada categoría individual considerada. De este modo, sabemos que el 59.1\% de los estudiantes con un CSF adecuado tienen un rendimiento académico básico y ninguno dentro de esta categoría tiene un rendimiento superior. Por otra parte, la mayoría de los estudiantes con un CSF inadecuado pertenecen a la modalidad de rendimiento académicos básico con un 58.8\%. Mientras que el menos frecuente es el rendimiento bajo con un $5.9 \%$, como se puede apreciar en la . Donde se agrupa el CSF por nivel académico.

Agrupación CSF por Rendimiento colegio público 
Figura 3.

Gráfico de barras agrupadas del CSF por Nivel académico

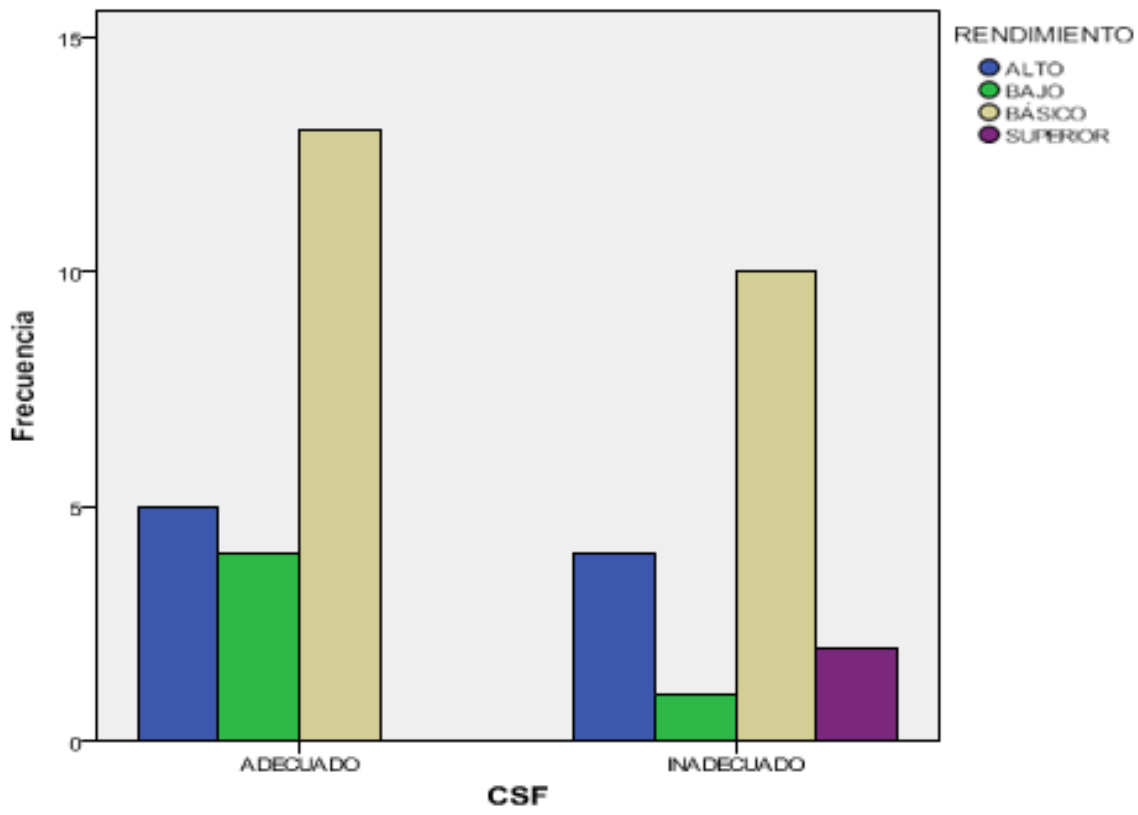

Dentro de los estudiantes con un rendimiento alto, el 55.6\% de ellos tienen un CSF adecuado contra un $44.4 \%$ con un CSF inadecuado. Por su parte, el total de los estudiantes con un rendimiento académico superior corresponden a la modalidad CSF inadecuado. El 80\% de los estudiantes con un rendimiento bajo tienen un CSF adecuado, y los estudiantes con un rendimiento básico se distribuyen entre un CSF adecuado y un CSF inadecuado, con el $56.5 \%$ y $43.5 \%$, respectivamente. De forma Gráfica esto se muestra en la figura 4 , donde el rendimiento académico se agrupa de acuerdo con las modalidades del CSF.

Figura 4.

Gráfico de barras agrupadas del Nivel académico por CSF.

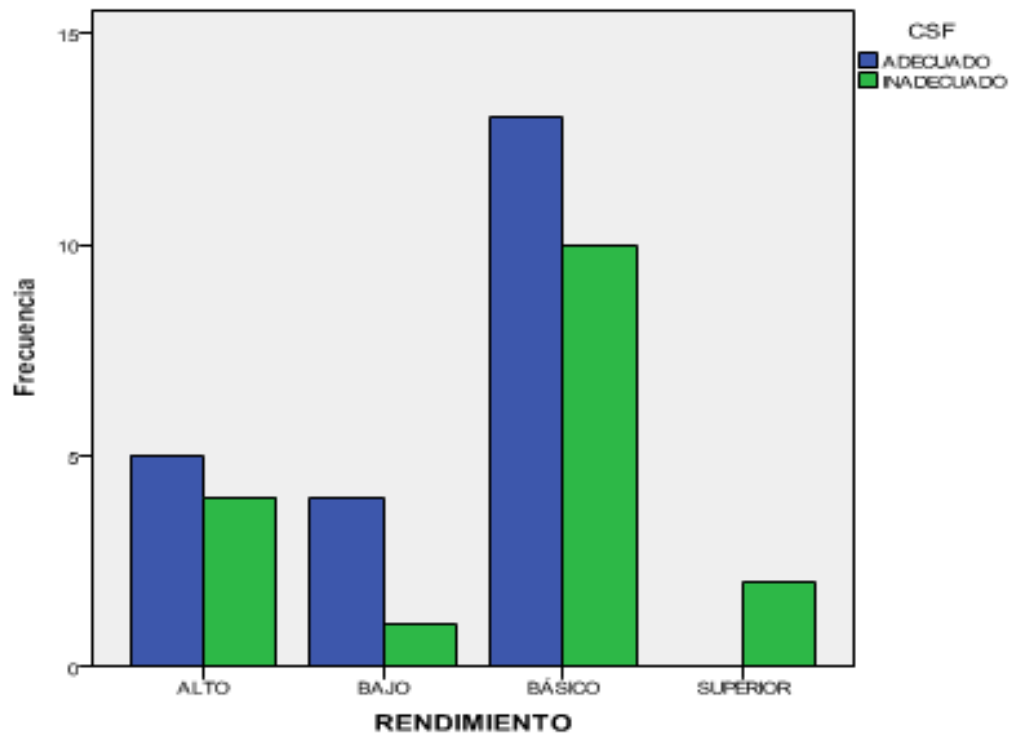


El análisis de la información contenida en la tabla de contingencia creada a partir de las variables clima social familiar y el rendimiento académico, se realizará con el uso de un modelo de regresión logística con ayuda del programa estadístico R2.14.1.
En este tipo de tablas habitualmente se desea conocer si existe asociación entre las dos variables, o si por el contrario se pueden considerar independientes.

Los datos del cuadro 2 de contingencias son reorganizados de la siguiente forma.

\begin{tabular}{|l|l|c|}
\hline CSF & RENDIMIENTO & FRECUENCIA (F) \\
\hline ADECUADO & ALTO & 5 \\
ADECUADO & BAJO & 4 \\
ADECUADO & BASICO & 13 \\
ADECUADO & SUPERIOR & 0 \\
INADECUADO & ALTO & 4 \\
INADECUADO & BAJO & 1 \\
INADECUADO & BASICO & 10 \\
INADECUADO & SUPERIOR & 2 \\
\hline
\end{tabular}

Modelo de regresión logística: Los modelos de regresión logística son modelos de regresión que permiten estudiar si una variable binomial depende, o no de otra u otras variables (no necesariamente binomiales). La variable respuesta binaria de interés en este caso es el CSF con dos posibles resultados: Adecuado e Inadecuado, y la variable independiente es el Rendimiento académico.

\section{Hipótesis de interés}

H0: existe relación entre las variables CSF y el rendimiento académico

Ha: no existe relación entre las variables CSF y el rendimiento académico

Tabla 3.

Coeficientes.

\begin{tabular}{|c|c|c|c|c|c|}
\hline \multicolumn{6}{|c|}{ Coefficients } \\
\hline & Estimate & Std. Error & & value $z$ & $\operatorname{Pr}(>|z|)$ \\
\hline (Intercept) & $\begin{array}{l}- \\
0.2231 \\
4\end{array}$ & 0.67082 & $\square$ & -0.333 & 0.739 \\
\hline RENDIMIENTO[T.BAJO] & $\begin{array}{l}- \\
1.1631 \\
5\end{array}$ & 1.30384 & $\square$ & -0.892 & 0.372 \\
\hline
\end{tabular}




\begin{tabular}{lclllll}
\hline RENDIMIENTO[T.BASICO] & $\begin{array}{l}\text { - } \\
\text { N.0392 }\end{array}$ & 0.79178 & -0.050 & 0.960 \\
& 2 & & & \\
& & & & \\
\hline RENDIMIENTO[T.SUPERIOR] & 17.12554 & 2007.45825 & 0.009 & 0.993 \\
\hline
\end{tabular}

Para un nivel de significancia de 0.05 y dado que el $\mathrm{P}$-valor $=0.2069>0.05$, no se cuenta con evidencia estadística suficiente para rechazar la hipótesis nula, por lo tanto, no existe asociación entre ambas variables.

\section{Estudio sobre el clima social familiar (CSF) y} el rendimiento académico en el colegio privado. A continuación, se presenta la información recolectada a través de cuestionario test de Moss (CSF).

Cuadro 5.

Datos para el clima social familiar y el rendimiento académico del colegio privado.

\begin{tabular}{|c|c|c|c|c|c|c|}
\hline ID & DR & $\mathrm{DD}$ & $\mathrm{DE}$ & TOTAL & CSF & RENDIMIENTO \\
\hline 1 & 16 & 30 & 14 & 60 & ADECUADO & ALTO \\
\hline 2 & 17 & 27 & 12 & 56 & ADECUADO & BASICO \\
\hline 3 & 13 & 22 & 14 & 49 & INADECUADO & ALTO \\
\hline 4 & 15 & 27 & 13 & 55 & ADECUADO & BASICO \\
\hline 5 & 16 & 27 & 13 & 56 & ADECUADO & BASICO \\
\hline 6 & 15 & 26 & 11 & 52 & ADECUADO & ALTO \\
\hline 7 & 16 & 31 & 12 & 59 & ADECUADO & BASICO \\
\hline 8 & 16 & 33 & 15 & 64 & ADECUADO & BASICO \\
\hline 9 & 13 & 35 & 14 & 62 & ADECUADO & BASICO \\
\hline 10 & 15 & 33 & 13 & 61 & ADECUADO & BASICO \\
\hline
\end{tabular}

A partir del cuadro 6 se construye la tabla de contingencias para las variables CSF y Rendimiento académica donde para efectos de análisis solo se consideran las modalidades alto y básico dado que para las otras modalidades son nulas. De esta forma se obtiene una tabla $2 \times 2$.

Cuadro 6.

Recuento.

Recuento

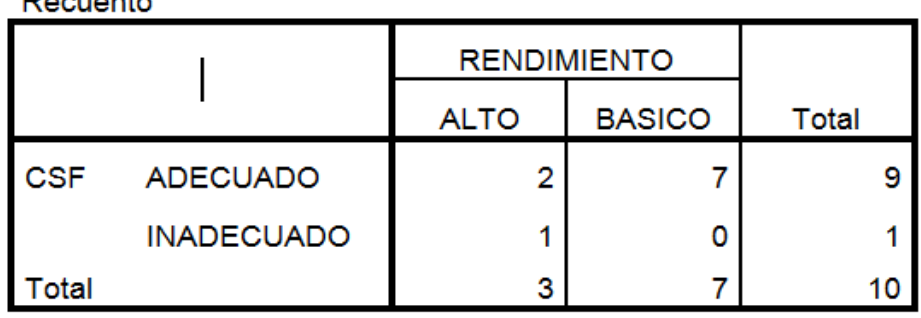


Cuadro 7.

Tabla de contingencia CSF por rendimiento.

\begin{tabular}{|c|c|c|c|c|c|}
\hline & & & \multicolumn{2}{|c|}{ RENDIMIENTO } & \multirow[b]{2}{*}{ Total } \\
\hline & & & ALTO & BASICO & \\
\hline \multirow[t]{8}{*}{ CSF } & ADECUADO & Recuento & 2 & 7 & 9 \\
\hline & & $\%$ dentro de CSF & $22,2 \%$ & $77,8 \%$ & $100,0 \%$ \\
\hline & & $\%$ dentro de RENDIMIENTO & $66,7 \%$ & $100,0 \%$ & $90,0 \%$ \\
\hline & & $\%$ del total & $20,0 \%$ & $70,0 \%$ & $90,0 \%$ \\
\hline & INADECUADO & Recuento & 1 & 0 & 1 \\
\hline & & $\%$ dentro de CSF & $100,0 \%$ &, $0 \%$ & $100,0 \%$ \\
\hline & & $\%$ dentro de RENDIMIENTO & $33,3 \%$ &, $0 \%$ & $10,0 \%$ \\
\hline & & $\%$ del total & $10,0 \%$ &, $0 \%$ & $10,0 \%$ \\
\hline \multirow[t]{4}{*}{ Total } & & Recuento & 3 & 7 & 10 \\
\hline & & $\%$ dentro de CSF & $30,0 \%$ & $70,0 \%$ & $100,0 \%$ \\
\hline & & $\%$ dentro de RENDIMIENTO & $100,0 \%$ & $100,0 \%$ & $100,0 \%$ \\
\hline & & $\%$ del total & $30,0 \%$ & $70,0 \%$ & $100,0 \%$ \\
\hline
\end{tabular}

Del cuadro 7 se observa que el $77.8 \%$ de las personas que tienen un CSF adecuado presentan un rendimiento académico básico, pero de aquellas que tienen este mismo rendimiento académico ninguna pertenece a un CSF inadecuado. Por lo tanto, el total de los estudiantes con un rendimiento básico tienen un CSF adecuado como se aprecia en el cuadro 7. Además, el total de los estudiantes con un CSF inadecuado presentan un rendimiento académico alto como se indica en el cuadro 7.

Figura 5.

Gráfico de barras agrupadas del Rendimiento académico por CSF colegio privado.

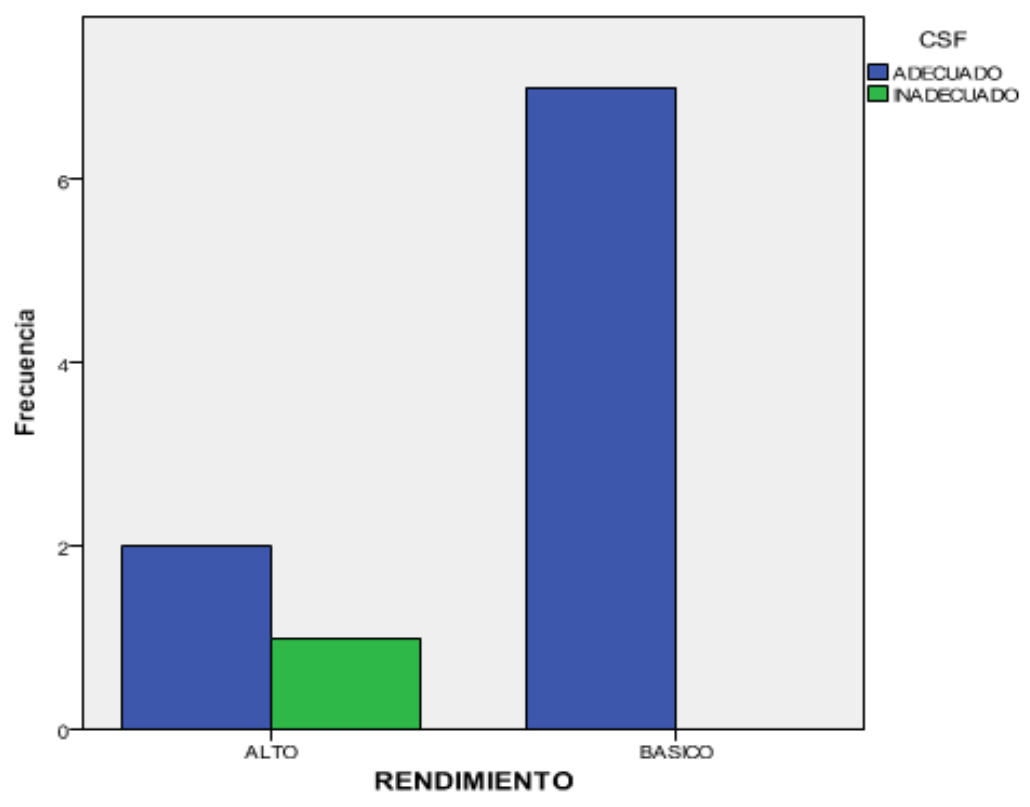



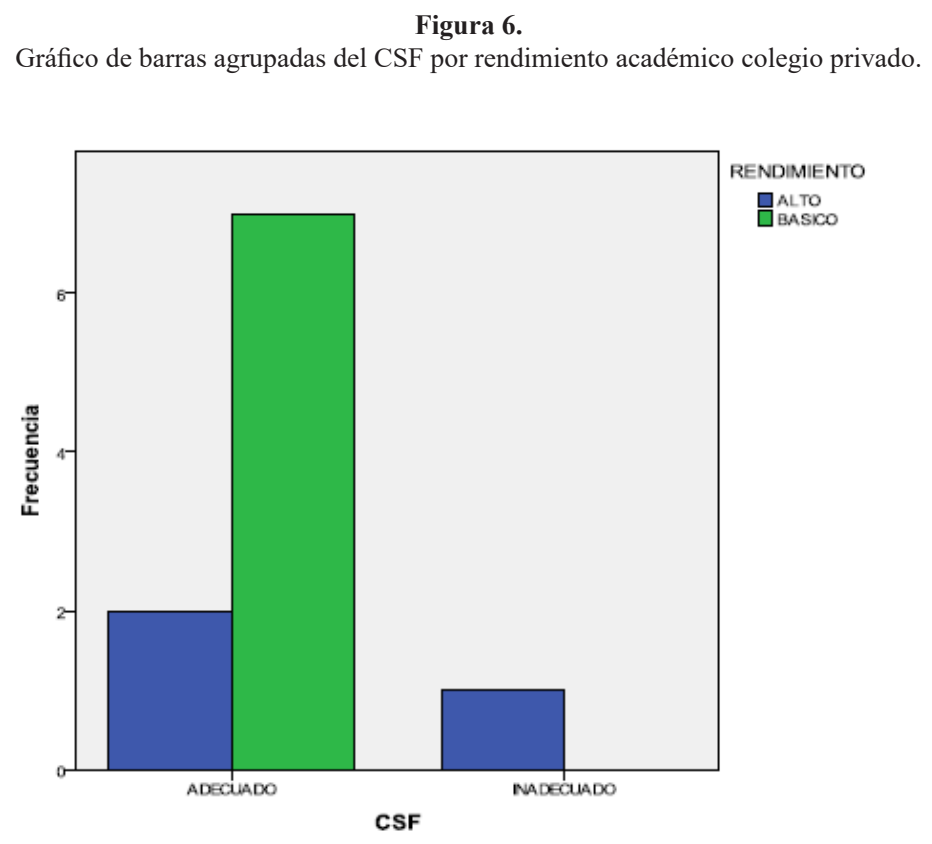

\section{Test exacto de Fisher}

El test exacto de Fisher permite analizar si dos variables dicotómicas están asociadas cuando la muestra a estudiar es demasiado pequeña y no se cumplen las condiciones necesarias para que la aplicación del test chi-cuadrado sea adecuada. Este test se basaen evaluar la probabilidad asociada a cada una de las tablas $2 \times 2$ que se pueden formar manteniendo los mismos totales de filas y columnas que los de la tabla observada. Cada una de estas probabilidades se obtiene bajo la hipótesis nula de independencia de las dos variables que se están considerando.

El cuadro 2 de contingencias para estudiar las diferencias en el rendimiento académico entre las dos modalidades del CSF, para estos 10 estudiantes.

La probabilidad exacta de observar un conjunto concreto de frecuencias a, b, c y d en una tabla $2 \mathrm{x}$ 2 cuando se asume independencia y los totales de filas y columnas se consideran fijos viene dada por la distribución hipergeométrica:

$$
P=\frac{(a+b) !+(c+d) !+(a+c) !(b+d) !}{n ! a ! b ! c ! d !}
$$

Esta fórmula se obtiene calculando todas las posibles formas en las que podemos disponer $n$ sujetos en una tabla $2 \times 2$ de modo que los totales de filas y columnas sean siempre los mismos, $(\mathrm{a}+\mathrm{b})$, $(\mathrm{c}+\mathrm{d}),(\mathrm{a}+\mathrm{c})$ y $(\mathrm{b}+\mathrm{d})$.

Este valor de $\mathrm{p}$ indicará la probabilidad de obtener una diferencia entre los grupos mayor o igual a la observada, bajo la hipótesis nula de independencia.
Si esta probabilidad es pequeña $(\mathrm{p}<0.05)$ se deberá rechazar la hipótesis de partida y deberemos asumir que las dos variables no son independientes, sino que están asociadas. En caso contrario, se dirá que no existe evidencia estadística de asociación entre ambas variables. 
Hipótesis

H0: el rendimiento académico es independiente del CSF

Ha: el rendimiento académico no es independiente del CSF

El resultado obtenido con el procedimiento fisher. test() en el programa R es:

Fisher's Exact Test for Count Data

$\mathrm{p}$-value $=0.3$

alternative hypothesis: true odds ratio is not equal to 1

Para un nivel de significancia de 0.05, dado que el valor de $\mathrm{P}$ es 0.3 se concluye que no existe evidencia estadística de asociación entre el rendimiento académico y el clima social familiar.

\section{Conclusiones}

Por medio de la investigación relación del clima social familiar y el rendimiento académico se logró identificar que en la institución educativa pública, no existe una asociación del rendimiento académico y el clima social familiar, ya que por medio de los resultados obtenidos del modelo de regresión logística se puede evidenciar que para un nivel de significancia de 0.05 y dado que el P-valor $=0.2069>0.05$, no se cuenta con evidencia estadística suficiente para rechazar la hipótesis nula, por lo tanto no existe asociación entre ambas variables.

Por otra parte en la institución educativa privado, por medio del el test exacto de Fisher, que permite analizar si dos variables dicotómicas están asociadas cuando la muestra a estudiar es demasiado pequeña y no se cumplen las condiciones necesarias para que la aplicación del test chi-cuadrado sea adecuada, por lo tanto para un nivel de significancia de 0.05, dado que el valor de $\mathrm{P}$ es 0.3 se concluye que no existe evidencia estadística de asociación entre el rendimiento académico y el clima social familiar.
Para un nivel de significancia de 0.05 y dado que el $\mathrm{P}$-valor $=0.2069>0.05$, no se cuenta con evidencia estadística suficiente para rechazar la hipótesis nula, por lo tanto, no existe asociación entre ambas variables.

Por lo tanto, se corroboran las teorías de los diversos autores como Papalia, Wendkos y Dustin, (2005), Pérez, (1996); Polaino,(2004), quienes afirman que en el rendimiento académico no solo influye el adecuado clima familiar si no que parte de múltiples factores como lo es en el ámbito escolar, socio económico y factores personales.

Dentro de la aplicación del test de Moos a la muestra trabajada se encontraron resultados que permiten concluir que según estimación estadística la media no indica correlación entre las variables rendimiento escolar y clima social familiar en las dos instituciones.

Al realizar el análisis estadístico bajo el modelo de regresión logística con la ayuda del programa estadístico R2.14.1, se concluye que no existe asociación entre las dos variables, dado que el porcentaje de correlación es de 0.269 , lo cual no es significativo para indicar un grado de correlación, esto permite establecer que para identificar algunos fenómenos del ser humano se hace importante el abordarlo desde sus diferentes dimensiones.

Los resultados indican que el $43.50 \%$ de la muestra de la institución educativa pública, presentan un inadecuado clima social familiar en contrataste con un $10 \%$ de la institución educativa privada, marcando un índice elevado de alteraciones a nivel familiar que pueden estarse reflejando en otras áreas de los jóvenes.

Dentro de la interpretación de los resultados y haciendo la claridad que no existe correlación entre las variables los factores encontrados en el inadecuado clima social familiar son en la dimensión de las relaciones que es la que mide el grado de comunicación y libre expresión al interior de la familia y el grado de interacción conflictiva que la caracteriza. 
Los datos obtenidos en la aplicación del test de Moos en el colegio público arrojan que del 43.6\% de inadecuado clima social familiar el 5.6 $\%$ tiene una relación con el bajo rendimiento académico encontradose en este como factores psicosociales la sub escala de Actuación (AC): Grado en el que las actividades (tales como el Colegio o el Trabajo) se enmarcan en una estructura orientada a la acción-competencia esta subescala se encuentra ubicada en la dimensión de desarrollo que evalúa la importancia que tiene dentro de la familia, ciertos procesos de desarrollo personal, que pueden ser fomentados o no, por la vida en común.

El proceso investigativo condujo a establecer como necesidad el construir una cartilla que tiene como objeto informar a los agentes activos del proceso educativo para potencializar al joven $\mathrm{y}$ fortalecer su rendimiento académico.

El realizar comparación entre dos instituciones donde se trabaja bajo una implementación igual, permite concluir que factores ambientales $y$ educativos como condiciones socioeconómicas marcan diferenciación entre las dos, partiendo por los niveles de rendimiento académico como del mismo porcentaje de casos de clima social familiar inadecuado.

La afectación que tenga un joven en su rendimiento escolar no se puede generalizar sino esta debe ser vista en sus particularidades incluyendo factores ambientales como las condiciones de estudio del joven, las características del aula y en general la relación propia de este con su entorno.

Debido a que los resultados obtenidos de la aplicación del test de Moos (ESF), no mostraron una correlación significativa en cuanto a las variables propuestas, se toma la decisión de realizar una cartilla informativa dirigida a los agentes implicados en el proceso educativo, dando a conocer pautas que potencialicen el rendimiento académico de los estudiantes, debido a que la media del rendimiento académico se encuentra en un nivel básico.

\section{Referencias}

AdueJ.G.(1997). Factores ambientales que afectan el rendimiento escolar de los niños provenientesde familias de bajo nivel socioeconómico y cultural. Estud. pedagóg, 23,75-80. doi:10.4067/S071807051997000100007.

Anabalón, M; Carrasco, S., Díaz, D., Gallardo C. y Cárcamo, H. (2008). El compromiso familiar frente al desempeño académico de niños y niñas de educación general básica en la ciudad de Chillán. Horizontes Educacionales, 13(1), 1121.

Alcalay, L.; Milicic, N.y Torretti, A. (2005). Alianza Efectiva Familia -Escuela: Un Programa Audiovisual Para Padres. Psykhe, 14(2),149161. doi: 10.4067/S0718-22282005000200012.

Baeza S. (2000). El rol de la familia en la educación de los hijos. Publicación virtual de la Facultad de Psicología y Psicopedagogía de la USAL, $3,1-10$

Barudy, J. y Dantagnan, M. (2005). Los buenos tratos en la infancia. Barcelona: Editorial Gedisa

Beltrán, J.\& Bueno, J. (1995). Psicología de la educación. España: Ed. Boixareu Universitaria.

Bima, H. (1980). El mito de la dislexia. México: Ateneo Nuevo Mar.

CIDE \& UNICEF. (2000). Diálogo público: Participación de los centros de padres en la educación. Santiago: CIDE-UNICEF.

Cassullo, G.L.; Álvarez L. V. y Pasman, P. (1998). Adaptación de las escalas de clima social escolar y familiar, VI Anuario de Investigaciones, Facultad de Psicología, Universidad de Buenos Aires: 186-198.

Clavijo, A., Casas, R. y Osorio, M. (2005). Psicología Educativa. http://psicoloeducativa. blogspot.com.co/

Coll, C., Palacios, J, y Marchesi, A. (2007). Desarrollo Psicológico y Educación, Psicología de laEducación Escolar. Madrid: Alianza.

Dios, C. et al. (2003). Factores familiares y sociales asociados al bienestar emocional enadolescentes diabéticos.Eur. J. Psychiat. 
17(3), 171-182. doi: http://dx.doi.org/10.4321/ S1579699X2003000300005.

Espitia Carrascal, R. E. \& Montes R. (2009). Influencia de la familia en el proceso educativo de los menores del barrio costa azul de Sincelejo (Colombia). 17(1).

Fernández-Ballesteros, R. y Sierra, B. (1982). Estudio factorial sobre la percepción del ambiente escolar. En: Fernández-Ballesteros, R. (Dir.). Evaluación de contextos. Reunión Nacional sobre intervención Psicológica, Universidad de Murcia, España.

Gabriela, L. Algunas consideraciones acerca del concepto de clima social y su evaluación.

Garbanzo V. (2007). Factores asociados al rendimiento académico en estudiantes universitarios, una reflexión desde la calidad de la educación superior pública. Costa Rica.

García, C. (2005). Habilidades Sociales, Clima Social Familiar y Rendimiento Académico en Estudiantes Universitarios. Universidad de San Martín de Porres, Escuela Profesional de Psicología. ISSN: 1729 - 4827. Lima-Perú.

González P. \& Pereda I, (2006). Relación entre el clima social familiar y el rendimiento escolar de los alumnos de la institución educativa $\mathrm{N}^{\circ}$ 86502 "San Santiago de Pomaras. Perú.

González, M. (1999). La relación familia-escuela: Sobre los "actores invisibles" y los "libretos silenciados" en la educación. Revista de la Compañía de Jesús en el Uruguay, 51-54.

Hernández, M (2008) Los campos de acción del psicólogo educativo. México.

Mathiesen, M. E.; Mora, O.; Chamblas, I. y Navarro, Gracia. (2004). Familia, Permisividad y Juicio Moral en Estudiantesde Enseñanza Media de la Provincia de Concepción. Psykhe,13(1), 3-20. doi: $10.4067 / \mathrm{S} 0718-22282004000100001$.

Moos, R. (1974) The Social Climate Scale: An overview. Palo alto, California: Consulting Psychologists Press

Moos, R. H. y Trickett, E.J. (1974) Classroom Environment Scale Manual. Palo Alto, California: Consulting Psychologists Press
Moos, R. H. y Moos, B. S. (1981). Family Environment Scale Manual. Palo Alto, California: Consulting Psychologists Press.

Moos, R. H.; Moos, B.S. y Tricket, E.J. (1995). Escalas de Clima Social. Madrid: TEA Ediciones Moreno, D., Estévez E., Murgui S. y Musitu, G. (2009). Relación entre el clima familiar y el clima escolar: el rol de la empatía, la actitud hacia la autoridad y la conducta violenta en la adolescencia. International Journal of Psychology Therapy, 9(1), pp.123-136.

Musitu, G. y García, F. (2004). Consecuencias de la socialización familiar en la cultura española. Psicothema, 16, pp. 288-293.

Papalia, D., Wendkos, S. y Duskin, R. (2005). Psicología del desarrollo. México: McGrawHill.

Pérez S., A.M. (1996). Factores psicosociales y rendimiento académico (Tesis de doctorado). Alicante (España): Universidad de Alicante.

Pita, S. \& Pértegas, S. (2002). Investigación cuantitativa y cualitativa.

Polaino, A. (2004). Familia y autoestima. Barcelona: Ariel.

Rojas, L, (2005). Influencia del entorno familiar en el rendimiento académico de niños y niñas con diagnóstico de maltrato de la escuela de calarca. Ibagué.

Sánchez, E. \& Valdés, C. (2011). Una aproximación a la relación entre el rendimiento académico y la dinámica y estructura familiar en estudiantes de primaria. México.

Seisdedos, N., Victoria de la Cruz, M. y Cordero, A. (1989). Escalas de Clima Social (FES). Madrid: TEA Ediciones.

Torres V. y Rodríguez S. (2006). Rendimiento académico y contexto familiar en estudiantes universitarios. México.

Urbina, S. (2000). Programa de residencia en psicología ambiental. México.

Villarroel Rosende, G. y Sánchez Segura, X. (2002). Relación Familia y Escuela: un estudio comparativo en la ruralidad. Estud.pedagóg. 123141.doi: 10.4067/S0718-07052002000100007. 
Walters Pacheco, K. Z.; Cintrón Bou, F. N.; SerranoGarcía, I. (2008), Familia Reconstituida. El Significado de Familia en la Familia Reconstituida Psicología Iberoamericana.14(2), pp. 16-27 Universidad Iberoamericana, Ciudad de México, México. 